\title{
Performance Study of Node-Disjoint Multipath Routing in Vehicular Ad Hoc Networks
}

\author{
Xiaoxia Huang, Member, IEEE, and Yuguang Fang, Fellow, IEEE
}

\begin{abstract}
Many multipath routing schemes have recently been proposed to improve the performance of wireless networks. Multipath routing is supposed to reduce the end-to-end packet delay and increase the packet delivery ratio. Therefore, it can also improve the packet delivery ratio in vehicular ad hoc networks (VANETs) when the mobility of relaying vehicles is unknown. However, in wireless networks, multiple paths are exposed to mutual interference or path coupling, which impairs efficiency. The intriguing question is whether the node-disjoint multipath routing really helps. In this paper, we examine the performance of node-disjoint multipath routing in VANETs. Through extensive simulations, we explore the effect of mutual interference on the behavior of node-disjoint paths. It is shown that whether node-disjoint paths are able to improve performance, compared with the single path, is determined by path coupling and the source-destination distance. Results show that node-disjoint multipath routing can be applied to VANETs to substantially improve performance in terms of delay and packet delivery probability only if the node-disjoint paths are properly chosen.
\end{abstract}

Index Terms-Interference, redundancy, reliability, routing.

\section{INTRODUCTION}

$\mathbf{I}$ N WIRELESS networks, because of interference, path loss, and channel fading, the performance tends to degrade as the number of hops of a path increases. For example, the throughput quickly declines as packets traverse a long path [1], and the endto-end delay is prolonged because of processing, contention, and retransmissions. Moreover, the packet loss rate increases with the hop counts. In addition, unknown mobility of vehicles causes high unreachable possibility of packets. Researchers are attempting to improve performance in vehicular ad hoc networks (VANETs) using various kinds of techniques. Among these, multipath routing is regarded as a potential solution to improve end-to-end delay and packet delivery ratio in VANETs. When packets are simultaneously transferred over several paths

Manuscript received January 31, 2008; revised August 31, 2008. First published October 31, 2008; current version published April 22, 2009. This work was supported in part by the US National Science Foundation under Grants CNS-0721744 and DBI-0529012. The work of X. Huang was supported in part by the National High Technology R\&D Program (863 Program) of China under 2006AA01A114. The work of Y. Fang was supported in part by the 111 Project under Grant B08038. The review of this paper was coordinated by Dr. H. Jiang.

X. Huang is with the Shenzhen Institute of Advanced Technology, Chinese Academy of Sciences, Shenzhen 518067, China (e-mail: xx.huang@ sub.siat. ac.cn).

Y. Fang is with the Department of Electrical and Computer Engineering, University of Florida, Gainesville, FL 32611 USA, and also with the National Key Laboratory of Integrated Services Networks, Xidian University, Xi'an 710071, China (e-mail: fang @ece.ufl.edu).

Color versions of one or more of the figures in this paper are available online at http://ieeexplore.iee.org.

Digital Object Identifier 10.1109/TVT.2008.2008094 between the source-destination pair, the throughput and endto-end delay could be improved. The packet delivery ratio can also be enhanced if backup or secondary paths are established, aside from the primary path, which is usually of the best quality. Some work [2] improves reliability by combining multipath routing with coding schemes, such as multiple description coding. Due to these favorable features of multipath routing, many multipath routing schemes have been proposed in literature, including both disjoint and braided multipaths [3]. However, performance evaluations of many multipath routing algorithms are carried out only on the network level and have not taken the medium access control (MAC) protocol and the path coupling into consideration. Is multipath routing always effective in practice for VANETs? If the answer is "no," the occasions in which multipath routing fails and the reasons behind the failure should be discussed. Moreover, the degree to which the performance can be improved and the cost of utilizing multiple paths are important issues to be investigated. Many papers try to address the problem. However, the results are based on some ideal assumptions, leading to usually approximate results or results that are only of theoretical use. Practical problems regarding the efficiency of multipath routing and optimal path selection remain unresolved.

Intuitively, multipath routing is not always efficient in terms of delay, packet delivery probability, and power consumption. The efficiency of multipath routing depends on the construction and physical distribution of paths. Path selection decides the performance of the multipath routing. If the selected paths are independent of each other or cooperative, multipath routing could improve the performance. On the other hand, if paths compete for shared resources, performance may even degrade and be worse than that of single path routing. When a link or a node is located on several paths, severe flow contention inevitably occurs when the incoming traffic load is high. As a result, the shared node or link becomes a bottleneck. Thus, node-disjoint path routing protocols are proposed to avoid interference among paths. However, paths with no links or nodes in common may still interfere with each other due to the broadcasting nature of wireless communications. Multiple flows traversing a common area could contend for resources, thus resulting in worse performance than expected. Therefore, paths that are out of each other's interference zone could be utilized to improve the performance [4]. Unfortunately, such paths are not always available when a number of flows exist. In [5], Tang et al. analyzed the interference level of interflow and intraflow contention [6]-[9] through flow contention graphs; however, there is no direct result on the interference level of general wireless ad hoc networks. To the best of our knowledge, 
this is the first time that the interflow interference of nodedisjoint paths is thoroughly studied. Our goal is to obtain comprehensive understanding on the efficiency of node-disjoint multiple paths through extensive simulations to investigate whether multipath routing is suitable for VANETs. Comparing the packet delivery ratio, end-to-end delay, and energy consumption of node-disjoint multipath routing and single path routing, we attempt to find out an efficient multipath pattern. Our work serves as a decision guideline for efficient multipath routing design.

This paper is organized as follows: Section II summarizes previous work on multipath routing. Section III illustrates how two paths interfere with each other. Then, we present the extensive simulation results in Section IV. In Section V, we study and summarize the evaluation results. Finally, Section VI concludes the paper.

\section{RELATED WORK}

Previous work extends the Ad hoc On-demand Distance Vector (AODV) routing and dynamic source routing (DSR) protocols to their multipath versions, but a few of them consider path coupling in the routing protocol design. Split multipath routing [11] is proposed to build an alternative path that is maximally disjoint from the shortest delay path. Unfortunately, path interference is not considered. As an extension to AODV, AODV-Multipath (AODVM) [12] is able to establish multiple node-disjoint paths. Marina and Das proposed AOMDV [13] to compute loop-free and link-disjoint paths. Incorporating the path accumulation feature of DSR into the extension of AODV, the routing protocol in [14] discovers multiple node-disjoint paths with low overhead. Nasipuri et al. extended DSR to support multipath routing [15]. CHAMP [16], which is based on DSR, uses cooperative packet caching and shortest multipath routing to enhance robustness against link breakdowns. Raghunathan and Kumar [17] proposed a distributed loadadaptive multipath routing protocol that converges to the Wardrop equilibrium. When the protocol reaches the equilibrium, all established paths between the source and destination have the same delay, which is less than that of any unused paths. In [3], algorithms used to discover link-disjoint multiple paths and braided multiple paths are proposed. Based on the per-hop channel error rate, which is assumed to be constant across the entire network, the number of outgoing paths is determined to achieve the desired reliability [18]. To combat unreliability, Tsirigos and Hass employ diversity coding and distribute packets over multiple disjoint paths [19]. Compromising between the violation of delay constraint and computation complexity, Orda and Sprintson applied network flow algorithms to obtain two disjoint paths as an approximation to the optimal feasible paths [20]. Felemban et al. addressed both time and reliability constraints in [21]. However, all of the aforementioned works focus on the routing protocol design and do not consider interference, which plays a significant role in the performance of multipath routing. How much gain could be achieved by multipath routing, considering MAC layer interference, remains to be answered. Since interference is decided by many factors, including interflow contention, intraflow contention, MAC layer protocol, physical layer communication technique, etc., we try to explore this complicated problem through thorough simulations. Jain et al. [22] modeled the interference between neighboring nodes using a conflict graph and presented methods for computing the optimal throughput, given the network placement and traffic load. The problem of how interference affects the performance of multipath routing still remains unresolved.

\section{Path Interference}

In wireless networks, the performance of multipath routing is constrained by mutual interference or path coupling of the involved paths. Due to the inevitable contention at shared nodes on two paths, braided paths [3] are likely to experience more serious interference than node-disjoint paths. Thus, nodedisjoint path routing is more preferable, considering mutual interference. Although node-disjoint paths seem to work independently as they have no nodes in common, except the source and destination nodes, they are also exposed to potential contention [6]-[9]. Thereafter, we focus on studying the path coupling between node-disjoint paths. If a node on one path is in the transmission range of a node on the other path, contention occurs. At most one node is allowed to transmit or receive at any time in a neighborhood. Thus, severe contention would cause reduced throughput and longer delay. Take the typical topology in VANETs shown in Fig. 1(a) as an example. Here, we assume that nodes have the same transmission range. Nodes $a$ and $e$ are within the transmission range of each other; thus, they contend for the channel before data transmission. Likewise, nodes $b, c$, and $f$ will experience serious contention for transmission opportunity. Only when any node on one path is out of the interference range of any node on the other path are the two paths free of contention. As shown in Fig. 1(b), nodes $d$, $e$, and $f$ do not contend with nodes $a, b$, and $c$ on the other path. In this case, the node-disjoint paths are the most efficient since the channel is fully reused in different regions. On the other hand, if a number of nodes on one path contend with the nodes on the other path, the efficiency of node-disjoint paths is expected to degrade. Fig. 1(a) and (b) shows the typical interference pattern in VANETs. Partial or whole paths are either adjacent to each other, causing strong coupling, or separated from each other without coupling. It is possible that node-disjoint paths with tight coupling perform poorly, compared to the single path, because severe collisions introduce substantial packet loss, delay, and energy consumption. Thus, packet delivery ratio, end-to-end delay, and energy consumption are the three metrics to be studied in the succeeding sections. In our paper, the packet delivery ratio is the ratio of the number of packets successfully received by the destination node to the total number of generated packets at the source node. It is actually a metric for measuring the packet delivery probability of using the selected paths.

In our simulation, two paths are used for packet delivery. Now, we want to show that the use of multiple paths can improve the packet delivery ratio if there is no interference. Let the packet delivery ratio for each single path be $P_{1}$ and $P_{2}$. Then, the packet delivery ratio that can be achieved through single path routing $P_{s}$ is

$$
P_{s} \leq \max \left\{P_{1}, P_{2}\right\} .
$$




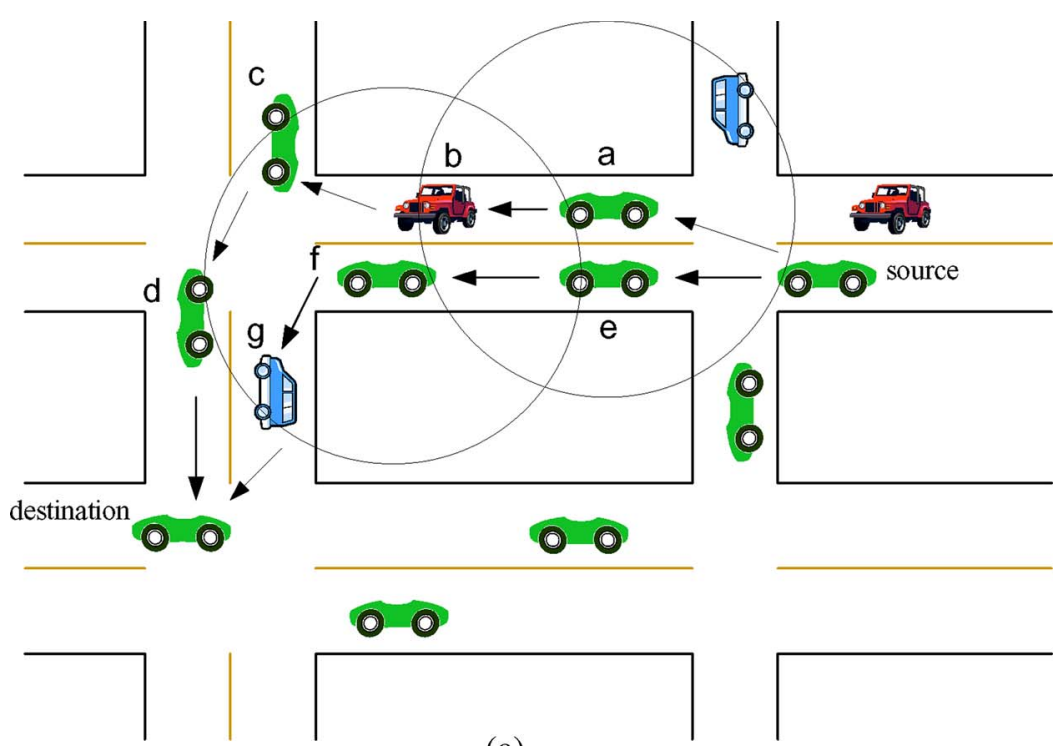

(a)

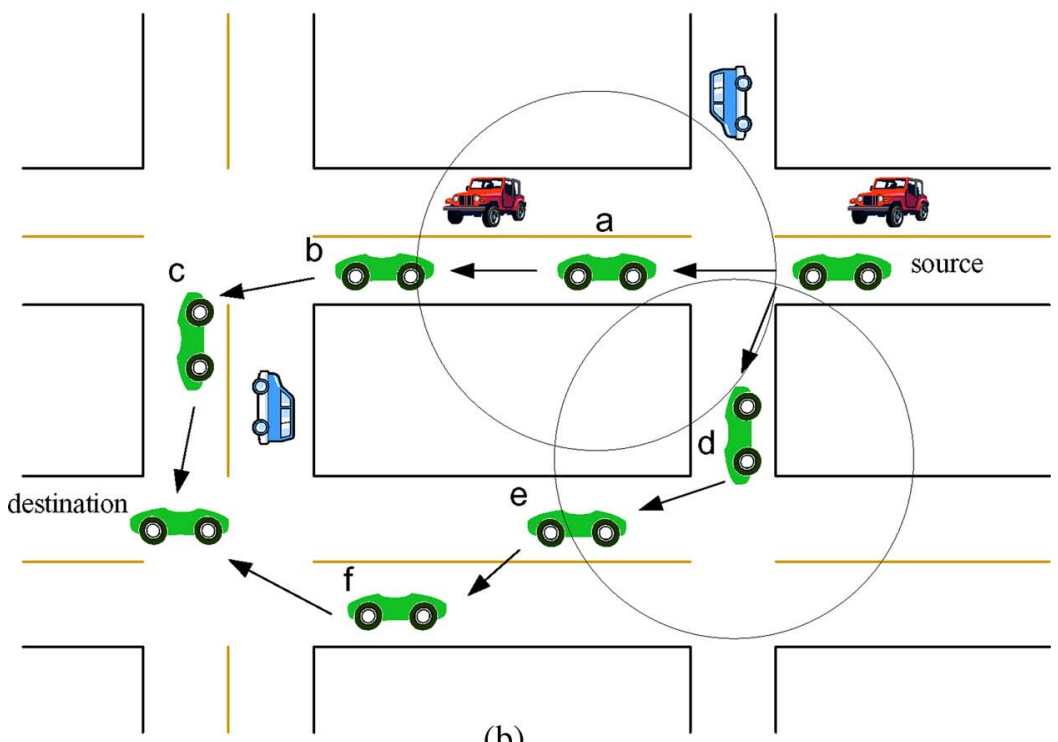

(b)

Fig. 1. Interference between node-disjoint paths. (a) Node-disjoint paths with coupling. (b) Node disjoint paths without coupling.

When using both paths for packet delivery, packet delivery ratio $P_{m}$ is

$$
P_{m}=1-\left(1-P_{1}\right)\left(1-P_{2}\right)=P_{1}+P_{2}-P_{1} P_{2} \text {. }
$$

Since $P_{1}, P_{2} \leq 1$

$$
P_{1} P_{2} \leq \min \left\{P_{1}, P_{2}\right\}
$$

We obtain

$$
P_{m} \geq \max \left\{P_{1}, P_{2}\right\} \geq P_{s}
$$

Obviously, multipath routing without path interference improves the packet delivery ratio, compared to single path routing.

However, path interference plays a significant role in disjoint multipath routing. A simplified model can be used to explain how path interference affects the aggregate reliability of disjoint multipath routing. Assume that the impact of path interference on the reliability of each path in the previous example can be characterized as $\alpha$ and $\beta$, where $0<\alpha, \beta \leq 1$. Then, the aggregate reliability when using both paths, which is denoted as $P_{\mathrm{mi}}$, is

$$
P_{\mathrm{mi}}=1-\left(1-\alpha P_{1}\right)\left(1-\beta P_{2}\right)=\alpha P_{1}+\beta P_{2}-\alpha \beta P_{1} P_{2} .
$$

When $\alpha=\beta=1, P_{\mathrm{mi}}=P_{m}$. This means that there is no path coupling between two paths. However, the relationship between $P_{\mathrm{mi}}$ and $P_{s}$ depends on $\alpha$ and $\beta$, which are difficult to obtain. From this model, we observe that the selection of node-disjoint paths, which decides $\alpha$ and $\beta$, is the key to the performance of node-disjoint multipath routing. Whether our result is valid when considering path interference or path coupling is to be investigated through simulations in the next section. 


\section{Performance Evaluation}

We evaluate the efficiency of node-disjoint multipath routing using simulator NS-2. Sixty nodes are randomly deployed in a $1000 \mathrm{~m} \times 1000 \mathrm{~m}$ square. The objective of this paper is to investigate the performance of node-disjoint paths subject to different levels of interference. To eliminate the coupling of the network performance and the underlying mobility model, we use the link error probability to indicate the packet loss due to multipath fading, shadowing, and Doppler shifts. The transmission range of each node is $250 \mathrm{~m}$, which is the same as the interference range. A source-destination pair is randomly selected in each run. Two node-disjoint paths between them are used for routing in the simulation. As our purpose is to study the mutual interference between node-disjoint paths, interflow contention becomes more apparent when only two interfering paths are present. To show the effect of path coupling on the performance of node-disjoint multipath routing, we compare its performance with shortest path routing. The widely used IEEE 802.11 is selected as the MAC layer protocol. The link rate is $2 \mathrm{Mb} / \mathrm{s}$. The queue at each node is able to hold at most 50 packets. The maximum MAC retransmission limit is seven for each packet.

\section{A. Path Selection}

First, we need to find out the node-disjoint paths between the given source and destination nodes $s$ and $d$ to examine the interference between them. To show the influence of interference on node-disjoint path routing, both the node-disjoint path pair with minimum coupling and that with maximum coupling are tested in the simulation. We say that two paths are coupled if any node, aside from the source and destination nodes, on one path is the neighbor of any node on the other path. A neighboring node is defined as a node within the transmission range. The two paths with minimum coupling have the fewest nodes interfering with the nodes on the other path, whereas the most serious coupling occurs if two paths have the most interfering nodes among all node-disjoint path pairs.

For single path routing, we choose the shortest path between the source and the destination. As the distance increases, the end-to-end packet delivery ratio quickly drops, and the delay increases. Therefore, the shortest path achieves the best performance in terms of delay. The $s-d$ distance is defined as the hop count of the shortest path between the source and destination nodes.

\section{B. Routing Without Redundancy}

1) Traffic Load: The selected source node splits and distributes the generated constant bit rate traffic $T$ over the two node-disjoint paths. Every path is offered approximately the same amount of packets, so the traffic load on each path is $T / 2$. The source node distributes the data packets to each path with a probability of $1 / 2$ whenever it generates a packet. When $T$ is large enough, as in our simulation, both paths are generally offered the same amount of packets. In the simulation, $T$ increases from 1 to 100 packets/s. Each data packet has a length of $512 \mathrm{~B}$.
2) Simulation Result: To assess the performance gain of multipath routing, we compare the simulation result of nodedisjoint multipath routing with that of single path routing. Since the performance sharply degrades as the hop count of a path increases, the simulation result considerably differs for source-destination pairs with different distances. Hence, the simulation results are grouped according to different $s-d$ distances. In our simulations, the $s-d$ distance varies from two to seven hops. The shortest path is selected in single path routing. For node-disjoint path routing, we expect that two paths far apart have slight mutual interference or even no mutual interference. Those paths are probably longer than the shortest path, because they widely separate in space. However, the simulation results show that this is not always true, because, sometimes, node-disjoint paths with more hops perform poorly, compared to shorter node-disjoint paths. The underlying reason is that both path length and path coupling affect the performance. Longer paths spread out farther away, resulting in mild or no coupling. However, a longer path consisting of more lossy or unpredictable wireless links suffers from relatively heavier packet loss and intraflow contention. Whether a widely dispersed node-disjoint path pair outperforms relatively close node-disjoint paths depends on the impact of both factors.

We compare the packet delivery ratio, end-to-end delay, and energy consumption of node-disjoint multipath routing and single path routing. For each $s-d$ distance, the reported result is averaged over seven runs. To verify the efficacy of multipath routing in VANETs with uncertain node mobility, we also compare the performance of multipath routing and single path routing when links are unreliable. We use link error probability to characterize the packet loss. Fig. 2 and Tables I and II also show the packet delivery ratio and end-to-end delay when the link error probability is 0.1 and 0.2 , respectively.

Fig. 2 shows the end-to-end packet delivery ratio for source and destination nodes with different distances. As the measurements have a similar trend for $s-d$ pairs with small variations in distance, we only show the performance of three-, five- and seven-hops-away $s-d$ pairs. The packet delivery ratio is the ratio of the number of packets received by the destination node to the total number of generated packets at the source node. It is actually a metric for measuring the packet delivery probability of using the selected paths. For short distance $s-d$ pairs, as shown in Fig. 2(a), node-disjoint path routing achieves almost the same packet delivery ratio as single path routing under all link conditions, because intraflow contention dominates. The advantage of node-disjoint path routing becomes more obvious as the $s-d$ distance increases. As shown in Fig. 2(c), the packet delivery ratio of the best node-disjoint paths approximately doubles that of the single path as the packet rate increases to more than 40 packets/s. For long distances such as seven hops, node-disjoint multipath routing outperforms single path routing, even in the worst situation. The worst node-disjoint paths achieve almost the same packet delivery ratio as the single path, because the path diversity of using two paths counteracts the path interference. Fig. 2(b) evidently shows how interference affects the performance of node-disjoint paths. The best nodedisjoint path pair performs better than that of the single path. However, the packet delivery ratio of the worst node-disjoint 


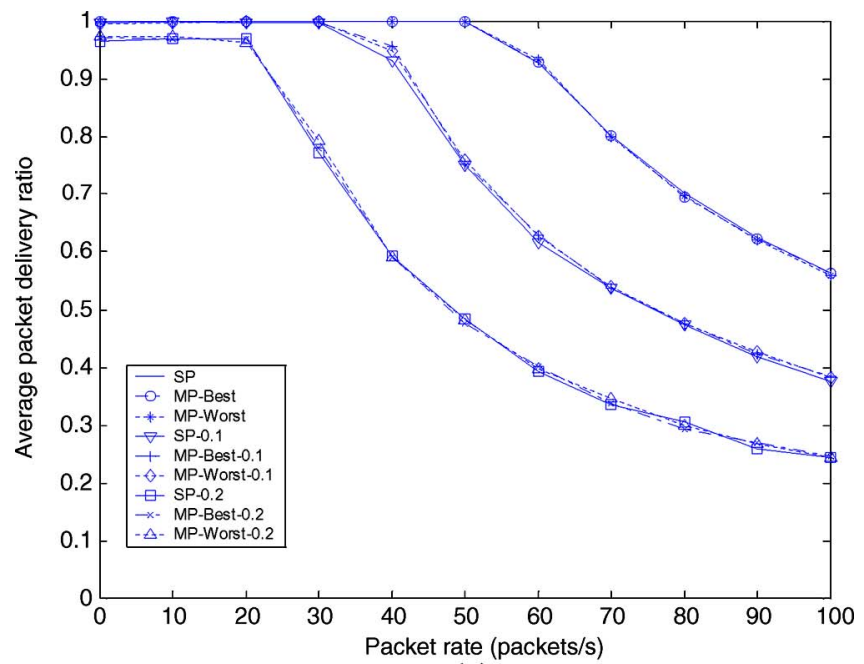

(a)

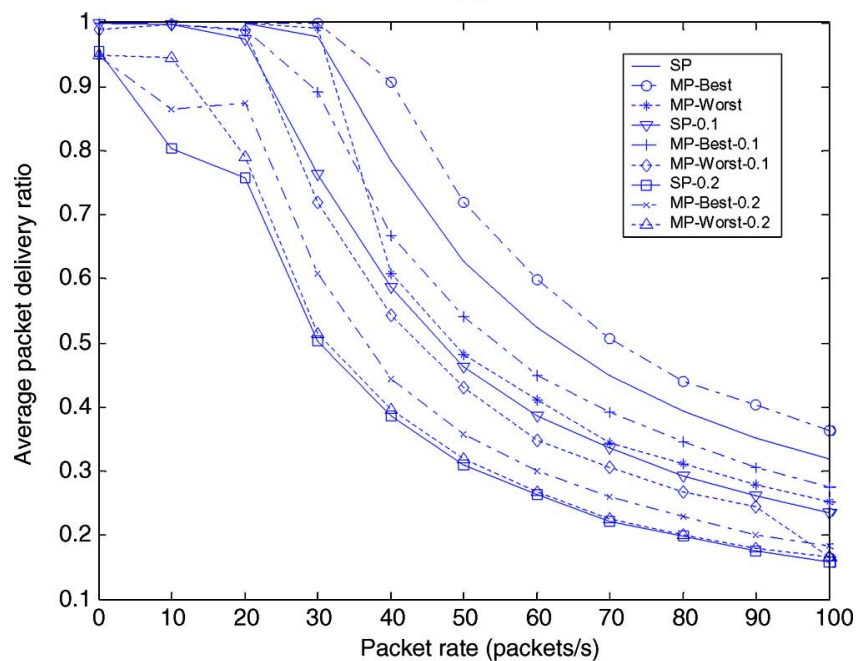

(b)

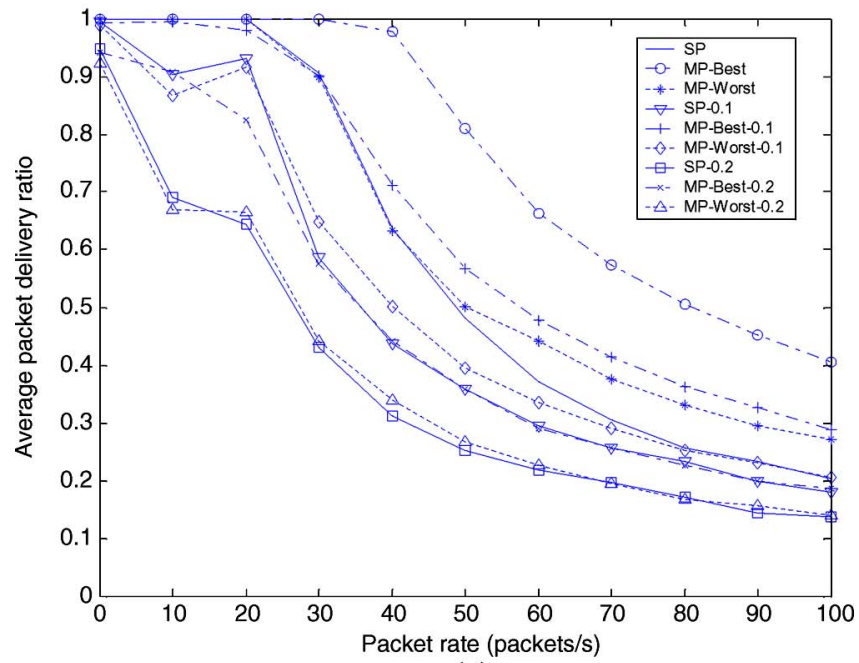

(c)

Fig. 2. Average end-to-end packet delivery ratio for different $s-d$ distances (a) 3-hop. (b) 5-hop. (c) 7-hop.

paths is lower than that of the single path, particularly when the packet rate is low. The packet delivery ratio of the worst nodedisjoint paths approaches that of the single path as the packet rate becomes greater, as shown in Fig. 2(b). When the links become lossy, the packet delivery ratio is still close to 1 when the packet rate is low. The reason is that retransmissions at the MAC layer can offset the link loss under light load. When the traffic load is heavy, packets are dropped as the queue is quickly filled up. The average waiting time increases because of the retransmission and queuing delay. Therefore, the service time is much longer than the packet arrival interval. Consequently, queue overflow happens, resulting in low packet delivery ratio.

The packet delivery ratio shows that the efficiency of nodedisjoint paths depends on both path coupling and path length. The efficiency of the node-disjoint path falls into three categories. For short distances, such as two and three hops in our simulation, node-disjoint paths achieve the same performance as the single path, because node-disjoint paths do not significantly diverge from the shortest path. For distances of four and five hops, the performance of node-disjoint paths greatly varies according to the degree of path coupling. In the best case, node-disjoint paths slightly outperform the single path, whereas node-disjoint paths poorly behave, compared to the single path in the worst case. For longer distances, e.g., seven hops, node-disjoint paths improve the performance, in terms of packet delivery ratio, compared to the single path no matter how serious two paths couple with each other. The best nodedisjoint paths outperform the single path by approximately $100 \%$ for seven-hop $s-d$ pairs when the packet rate is more than 40 packets/s. The worst node-disjoint paths achieve a packet delivery ratio comparable to that of the single path.

Tables I and II demonstrate the average end-to-end delay with respect to the packet rate when the source-destination pair is three and seven hops away, respectively. The average end-to-end delay increases as the $s-d$ distance increases in general. However, the end-to-end delay of the node-disjoint paths increases much more slowly than that of the single path. Again, the node-disjoint paths and the single path achieve comparable performances in terms of end-to-end delay when the source and destination nodes are only two or three hops away, as shown in Table I. Table II shows that the mostcoupled node-disjoint paths deliver the packets with the largest delay among the three when the link error probability is low. Relatively reliable links transport more packets, thus causing severe interflow contention due to path coupling. In Table II, the best node-disjoint paths attain the lowest average delay, because the node-disjoint paths are far away enough to transfer packets with less contention and offset the retransmission delay due to lossy links. However, in the worst case, node-disjoint paths suffer from serious contention due to path coupling. This causes the longest delay when links are error free. However, when link error is present, the worst node-disjoint paths deliver packets with shorter delay than the single path because of the advantage of load balancing.

Fig. 3 shows the average energy consumption for delivering a bit to the destination. The energy consumption per bit does not vary much for different packet rates, so we only compare them for different $s-d$ distances. As the link error probability increases, the average number of transmissions for a packet increases. Therefore, the average energy consumption increases as expected. The average energy cost is lower or comparable for node-disjoint paths, compared to the single path, when the 
TABLE I

Average END-TO-END DELAY WhEN $s-d$ distance $=3$

\begin{tabular}{|c|c|c|c|c|c|c|c|c|c|}
\hline & \multicolumn{3}{|c|}{ error-free } & \multicolumn{3}{|c|}{ error probability $=0.1$} & \multicolumn{3}{|c|}{ error probability $=0.2$} \\
\hline packets/s & SP & MP-Best & MP-Worst & SP & MP-Best & MP-Worst & SP & MP-Best & MP-Worst \\
\hline 1 & 0.017797 & 0.017781 & 0.017782 & 0.024236 & 0.023834 & 0.0242 & 0.035904 & 0.036929 & 0.3649 \\
\hline 20 & 0.01778 & 0.017779 & 0.017779 & 0.026235 & 0.026315 & 0.02592 & 0.10985 & 0.091922 & 0.09658 \\
\hline 40 & 0.018979 & 0.018852 & 0.018844 & 1.3183 & 1.2183 & 1.2559 & 2.5421 & 2.1787 & 2.1735 \\
\hline 60 & 0.8667 & 0.87468 & 0.86074 & 1.5074 & 1.4501 & 1.4361 & 2.4483 & 2.1983 & 2.2138 \\
\hline 80 & 0.90918 & 0.90861 & 0.90667 & 1.5148 & 1.4363 & 1.4928 & 2.5407 & 2.3115 & 2.2012 \\
\hline 100 & 0.91449 & 0.91668 & 0.91035 & 1.5657 & 1.4858 & 1.4972 & 2.395 & 2.1969 & 2.1784 \\
\hline
\end{tabular}

TABLE II

Average End-TO-End Delay When $s-d$ distance $=7$

\begin{tabular}{|c|c|c|c|c|c|c|c|c|c|}
\hline & \multicolumn{3}{|c|}{ error-free } & \multicolumn{3}{|c|}{ error probability $=0.1$} & \multicolumn{3}{|c|}{ error probability $=0.2$} \\
\hline packets/s & $\mathrm{SP}$ & MP-Best & MP-Worst & $\mathrm{SP}$ & MP-Best & MP-Worst & $\mathrm{SP}$ & MP-Best & MP-Worst \\
\hline 1 & 0.041843 & 0.041909 & 0.041907 & 0.057497 & 0.056907 & 0.05705 & 0.084095 & 0.084399 & 0.08472 \\
\hline 20 & 0.042236 & 0.041912 & 0.042743 & 0.24839 & 0.081952 & 0.19651 & 5.4833 & 0.55306 & 5.434 \\
\hline 40 & 2.4445 & 0.22801 & 4.5739 & 4.9595 & 3.5191 & 5.8782 & 7.3735 & 3.4184 & 5.8977 \\
\hline 60 & 2.6375 & 3.1191 & 4.5372 & 5.3574 & 3.3696 & 5.1944 & 7.5045 & 3.1942 & 4.9383 \\
\hline 80 & 3.398 & 3.1798 & 4.4164 & 4.9758 & 3.3871 & 4.7326 & 6.9673 & 3.0738 & 4.8928 \\
\hline 100 & 3.6611 & 3.156 & 4.217 & 5.5104 & 3.3462 & 5.1002 & 6.8799 & 3.3687 & 5.7538 \\
\hline
\end{tabular}

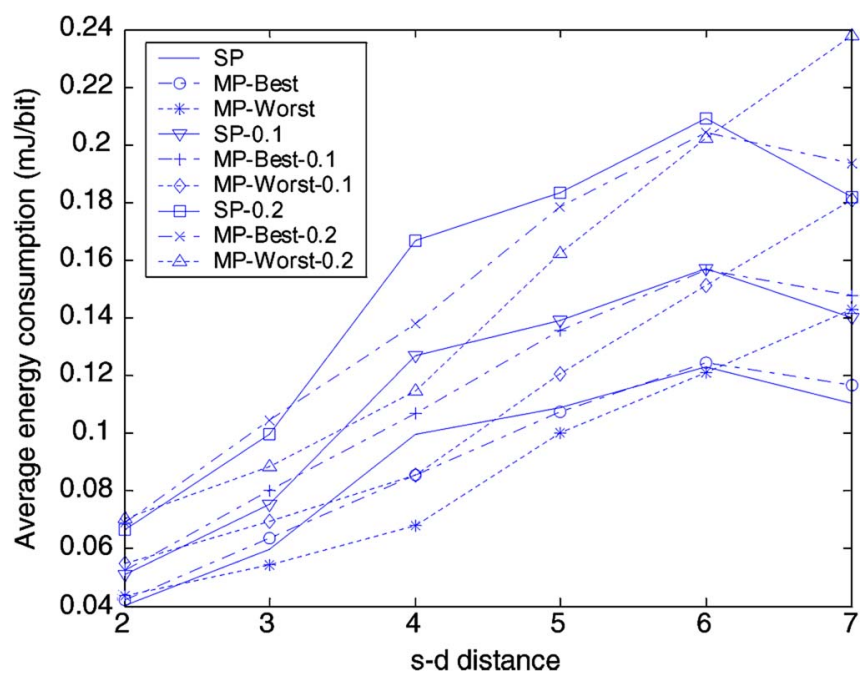

Fig. 3. Average energy consumption per bit for different $s-d$ distances.

$s-d$ distance is less than six hops. Among the three, the mostcoupled paths have the lowest average energy consumption per bit, because more packets are dropped at the first few hops; thus, collisions and transmissions are reduced. However, for seven-hops-away $s-d$ pairs, the average energy consumption per bit of node-disjoint paths is higher than that of the single path. Remember that both the most- and least-coupled nodedisjoint paths deliver more packets to the destination than the single path, so intraflow contention is more serious for them as the traffic becomes much heavier. This explains why the mostcoupled node-disjoint paths incur the highest energy cost for packet delivery when the source and destination nodes are far from each other, particularly when the link error probability is high.

\section{Routing With Redundancy}

In the previous simulation setting, every packet is only transferred over one of the two node-disjoint paths. If both two paths are used to deliver each packet, the packet delivery ratio is expected to be improved. In this simulation, we use both paths to deliver each packet. Once a source node generates a new packet, it simply distributes the packet to both paths. Apparently, the offered network traffic is doubled, compared to the previous setting. If a copy of the packet is correctly received by the destination from any of the two paths, it is counted as a successful delivery. Due to the redundancy, the same packet may arrive at the destination through both paths. Thus, the metric definitions are distinguished from the previous definitions and need to be redefined. The packet delivery ratio is the ratio of the total number of unique packets received at the destination to the total number of generated packets. The end-to-end delay of a received packet is the elapsed time before the successful reception of the first copy if two copies are received. The later received copy is simply discarded. The energy consumption per bit is the ratio of the total amount of consumed energy to the total number of received unique bits. Again, only bits in nonduplicate packets are counted.

As shown in the figures, the packet delivery ratio gains further improvement, compared to the scenario without redundancy. Packet delivery over both the best and worst nodedisjoint paths shows considerable enhancement of the packet delivery ratio, compared to that in the single path in all scenarios. For seven-hop $s-d$ pairs, the best node-disjoint paths deliver twice the number of packets than single path when the link error probability is 0.2 , whereas the worst node-disjoint paths achieve 50\% improvement over the single path. The performance gap between the best and worst node-disjoint paths becomes significant as the path length increases. When the $s-d$ pairs are just three hops away, the best and worst node-disjoint paths achieve comparable packet delivery ratio. However, when the $s-d$ distance increases to seven hops, the best node-disjoint paths gain as much as $40 \%$ higher packet delivery ratio than the worst node-disjoint paths. The underlying reason is that interflow contention dominates the performance. This explicitly shows that path coupling determines the efficiency of nodedisjoint multipath routing.

The end-to-end delay of the single path is the highest when the $s-d$ distance is short, as shown in Table III. When the $s-d$ distance is long and the packet rate is not very high, i.e., below 
TABLE III

Average End-To-End Delay When $s-d$ distance $=3$

\begin{tabular}{|r|c|c|c|c|c|c|c|c|c|}
\hline \hline & \multicolumn{3}{|c|}{ error-free } & \multicolumn{3}{c|}{ error probability=0.1 } & \multicolumn{3}{c|}{ error probability $=0.2$} \\
\hline packets/s & SP & MP-Best & MP-Worst & SP & MP-Best & MP-Worst & SP & MP-Best & MP-Worst \\
\hline 1 & 0.017797 & 0.008924 & 0.008924 & 0.024236 & 0.012367 & 0.012184 & 0.035904 & 0.018188 & 0.017853 \\
\hline 20 & 0.01778 & 0.00889 & 0.00889 & 0.026235 & 0.013197 & 0.013092 & 0.10985 & 0.043167 & 0.044065 \\
\hline 40 & 0.018979 & 0.009443 & 0.009442 & 1.3183 & 0.66609 & 0.6685 & 2.5421 & 1.3797 & 1.3766 \\
\hline 60 & 0.8667 & 0.4787 & 0.47221 & 1.5074 & 0.86921 & 0.86735 & 2.4483 & 1.5598 & 1.5154 \\
\hline 80 & 0.90918 & 0.6085 & 0.61088 & 1.5148 & 0.98557 & 0.97079 & 2.5407 & 1.604 & 1.6667 \\
\hline 100 & 0.91449 & 0.67983 & 0.68185 & 1.5657 & 1.0654 & 1.068 & 2.395 & 1.7001 & 1.6974 \\
\hline
\end{tabular}

TABLE IV

Average END-TO-END DELAY When $s-d$ distance $=7$

\begin{tabular}{|r|c|c|c|c|c|c|c|c|c|}
\hline \hline & \multicolumn{3}{|c|}{ error-free } & \multicolumn{3}{c|}{ error probability $=0.1$} & \multicolumn{3}{c|}{ error probability $=0.2$} \\
\hline packets/s & SP & MP-Best & MP-Worst & SP & MP-Best & MP-Worst & SP & MP-Best & MP-Worst \\
\hline 1 & 0.041843 & 0.020932 & 0.020931 & 0.057497 & 0.029404 & 0.028605 & 0.084095 & 0.044366 & 0.0453 \\
\hline 20 & 0.042236 & 0.02199 & 0.022227 & 0.24839 & 0.035835 & 0.09149 & 5.4833 & 0.24665 & 2.626 \\
\hline 40 & 2.4445 & 0.076197 & 3.1315 & 4.9595 & 2.3502 & 3.5323 & 7.3735 & 2.2608 & 3.7729 \\
\hline 60 & 2.6375 & 2.1201 & 3.4263 & 5.3574 & 2.6834 & 3.6029 & 7.5045 & 2.3254 & 4.0705 \\
\hline 80 & 3.398 & 2.3048 & 3.3605 & 4.9758 & 2.9516 & 3.6318 & 6.9673 & 2.3923 & 4.0436 \\
\hline 100 & 3.6611 & 2.4225 & 3.3299 & 5.5104 & 2.9708 & 3.9448 & 6.8799 & 2.6627 & 4.0225 \\
\hline
\end{tabular}

80 packets/s, the worst node-disjoint paths achieve the highest end-to-end delay, as demonstrated in the error-free column of Table IV. Although the shortest path is used in the single path case, retransmissions due to packet loss incur long delay. However, taking advantage of path diversity, a packet arrives at the destination with a shorter delay when using node-disjoint paths. The performance gain in terms of end-to-end delay becomes more significant as the path length or link error probability increases. The end-to-end delay achieved by the best nodedisjoint paths is $34 \%$ lower than that by the worst node-disjoint paths when the link error probability is 0.2 for seven-hops-away $s-d$ pairs. Since every packet is delivered over both paths, the average energy consumption per bit is much higher than that of the single path but less than twice. The energy cost of the best node-disjoint paths is the highest when the path length is less than seven hops, because they transport more packets than others. More retransmissions and more serious intraflow contention cause higher energy consumption. In short, the energy consumption is traded for improved packet delivery ratio and end-to-end delay.

Routing over two node-disjoint paths shows the same tendency as the previous setting. As the source and destination nodes get farther apart, the performance gains, in terms of packet delivery ratio and end-to-end delay, are more apparent. For example, the gain in the packet delivery ratio of nodedisjoint paths over the single path increases from approximately $40 \%$ to $110 \%$ as the $s-d$ distance increases from three to seven hops. Again, the most- and least-coupled node-disjoint paths achieve approximately the same performance at the short $s-d$ distance, i.e., three hops. As the $s-d$ distance increases, a difference in performance indicating the impact of the interference becomes apparent. The packet delivery ratio can be improved by up to $110 \%$ in Fig. 4(c). The end-to-end delay of the least-coupled node-disjoint paths increases at a much lower rate than the single path when the link error probability increases. As shown in Table IV, the end-to-end delay of the best and worst node-disjoint paths is up to $70 \%$ and $40 \%$ shorter than that of the single path, respectively. The disadvantage of node-disjoint paths is an increase of about $63 \%$ in energy consumption per bit in the worst situation for the least-coupled node-disjoint paths, as shown in Fig. 5. As the $s-d$ distance or link error probability increases, the energy consumption ratio of the best node-disjoint paths to the single path decreases. Thus, when the energy efficiency is not a strict requirement, which is usually true in VANETs, the use of the least-coupled nodedisjoint paths significantly improves the performance. The result justifies the use of carefully chosen node-disjoint paths with redundancy between the distant source and destination nodes in VANETs to improve the delay and packet delivery ratio.

\section{DISCUSSION}

Our performance evaluation studies reveal the relationship of path coupling and performance gain, and the relationship between the performance achieved by the node-disjoint paths and the $s-d$ distance. As the distance between the source and destination increases, the effectiveness of node-disjoint paths and the impact of path coupling become more remarkable.

The performance of node-disjoint paths and single path is indistinguishable for a close source-destination pair, i.e., two and three hops, when using one path for each packet. The reason is twofold: First, the node-disjoint path pairs are not widely spread, so they basically achieve comparable performance. Second, short paths assure short end-to-end delay, because the intraflow contention is not significant. Therefore, the buffer overflow at intermediate nodes is not caused by the large amount of delayed packets but the high packet rate. Both nodedisjoint path pair and single path attain the best performance achievable. However, transferring redundant packets over both paths improves the performance for all the $s-d$ distances, although the extent of improvement varies. The farther away the source-destination pair, the better the performance gain in packet delivery ratio and end-to-end delay achieved.

As the distance increases, node-disjoint path pairs disparately behave as the impact of path coupling becomes stronger. This is demonstrated by the performance gap of the best and worst node-disjoint paths in case of routing without redundancy. For four-hops-away $s-d$ pairs, the node-disjoint path pair without 


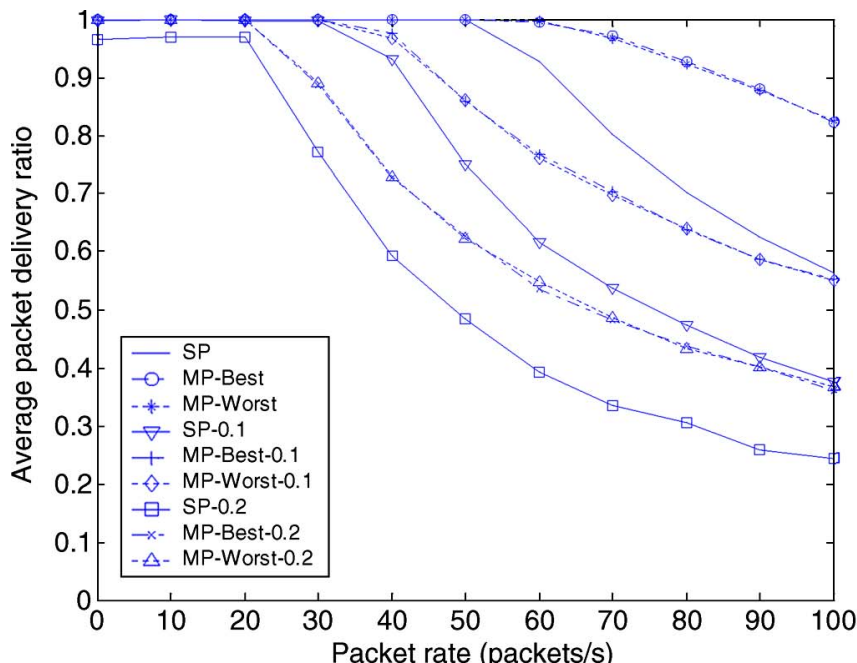

(a)

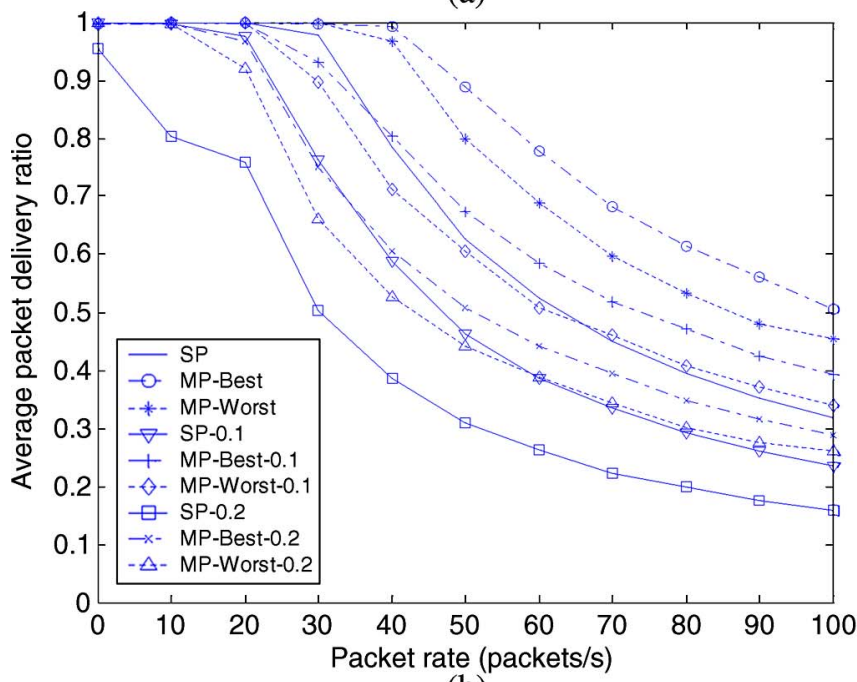

(b)

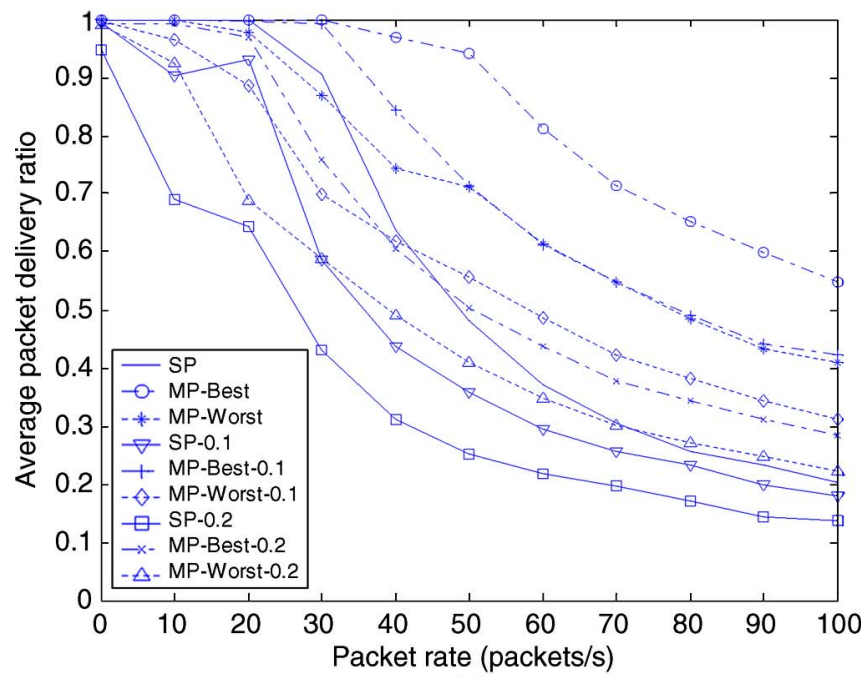

(c)

Fig. 4. Average end-to-end packet delivery ratio for different $s-d$ distances. (a) 3-hop. (b) 5-hop. (c) 7-hop.

packet redundancy outperforms the single path in the best case, particularly when the packet rate is high. On the contrary, the worst node-disjoint path pair performs worse than the single path in terms of packet delivery ratio. The packet delivery ratio

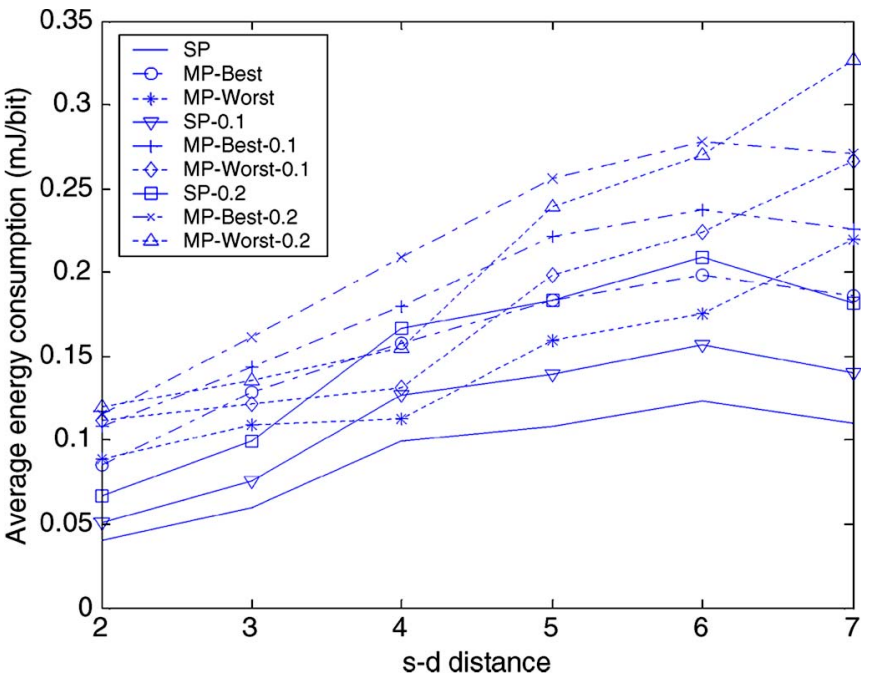

Fig. 5. Average energy consumption per bit for different $s-d$ distances.

of the two most-coupled paths is about $20 \%$ lower than that of the single path. The explanation for the different behaviors of node-disjoint path pairs is that interflow contention dominates. The least-coupled node-disjoint paths, which are widely separated, incur the lowest contention and perform best. They achieve the best packet delivery ratio with or without link errors. However, the most-coupled node-disjoint paths cause more serious contention than the single path. In addition to intraflow contention, they introduce interflow contention, which is absent from the single path. When link error is present, the enhanced packet delivery ratio of using two paths is explicitly shown in five-hops-away $s-d$ pairs.

For far-apart source-destination pairs, node-disjoint path routing generally shows advantage over single path routing. The performance gain in terms of packet delivery ratio of the node-disjoint path over the single path varies under different traffic loads. It is shown that the performance of node-disjoint path routing gracefully degrades, compared to that of single path routing when the network is congested. Moreover, nodedisjoint path pairs disparately behave in end-to-end delay with different $s-d$ distances. For the $s-d$ pair five or six hops away, the most-coupled node-disjoint paths attain the longest delay, whereas the single path achieves the shortest delay. For the seven-hop case, packets going through the least-coupled nodedisjoint paths experience the lowest end-to-end delay, whereas packets transferred over the most-coupled node-disjoint paths experience the longest delay when there is no link error. The phenomenon is attributed to the wider separation of longer paths, which further reduces the interflow contention of the best node-disjoint paths. Delivering more packets than the single path, the most-coupled node-disjoint paths incur more retransmissions due to collision, resulting in longer end-to-end delay. Retransmissions more seriously affect two interfering paths, because a single retransmission affects both paths, thus incurring heavier interflow contention. Therefore, the average packet delay of the worst node-disjoint paths is the largest of the three when the link is error free.

Some may expect multipath routing to be more energy consuming than single path routing, because more nodes are participating in communications. Our simulation shows that 
using two node-disjoint paths for each packet consumes more energy than the single path. However, if we just randomly use one of the two paths for every packet, the energy consumption per bit is lower than or close to that of the single path. There are two counteracting factors determining the energy consumption of node-disjoint path routing: One factor is the intraflow contention due to transmission, retransmission, collision, etc. Node-disjoint path routing is able to mitigate intraflow contention by distributing packets to two separated paths in the best situation. Another factor is the number of packets transported across the network. Node-disjoint paths enhance the packet delivery ratio and transfer more packets than the single path. The energy consumption of node-disjoint paths depends on which factor dominates.

Another observation is that the performance gap between the minimum-interference node-disjoint path pairs and the single path becomes conspicuous only at high packet rates. At a low packet rate, it is unlikely for many packets accumulating on a single path. Thus, the minimum-interference node-disjoint paths achieve basically the same performance as the single path without packet redundancy and moderate improvement in case of routing with redundancy. When the packet rate is high, a single path is more susceptible to congestion, and its performance abruptly degrades. The advantage of using node-disjoint paths is prominent with the distributed traffic load. From another perspective, a portion of the intense intraflow contention on the single path is substituted by the alleviated interflow contention between two node-disjoint paths with minimum mutual interference. The less coupling of the two paths, the less interflow contention, which results in better performance.

To sum up, it is more favorable to apply node-disjoint path routing for medium- to long-distance source and destination nodes at relatively high data rate if only one path is used for each packet. However, using both node-disjoint paths for every packet delivery significantly improves the performance if the pair of paths is cautiously picked. The drawback is the higher energy cost due to the higher traffic load across the network. Since energy is not a significant issue for vehicles, the higher packet delivery ratio and better end-to-end delay still justify the higher energy cost.

\section{CONCLUSION}

We have explored the efficiency of node-disjoint path routing subject to different degrees of path coupling, with and without packet redundancy. Simulation has validated that, through careful path selection, node-disjoint path routing gains an advantage over single path routing in terms of packet delivery ratio and end-to-end delay. The improvement is even more substantial when every packet is transferred over both paths. Since traffic is randomly distributed over chosen paths, the energy consumption of node-disjoint path routing without redundancy is lower than or comparable with that of single path routing. However, the energy consumption is higher than that of single path routing if both paths are used for packet delivery due to the much higher traffic load throughout the network.

Currently, no routing algorithm is capable of finding two minimum-interference paths between a source-destination pair in VANETs. Our future work is to design such a routing algorithm to improve end-to-end delay and packet delivery ratio in VANETs.

\section{REFERENCES}

[1] M. Haenggi and D. Puccinelli, "Routing in ad hoc networks: A case for long hops," IEEE Commun. Mag., vol. 43, no. 10, pp. 93-101, Oct. 2005.

[2] I. F. Diaz, D. Epema, and J. Jongh, "Multipath routing and multiple description coding in ad-hoc networks: A simulation study," in Proc. PE-WASUN, Oct. 2004, pp. 46-51.

[3] D. Ganesan, R. Govindan, S. Shenker, and D. Estrin, "Highly-resilient, energy-efficient multipath routing in wireless sensor networks," Mobile Comput. Commun. Rev. (MC2R), vol. 1, no. 2, pp. 251-254, 2002.

[4] S. Bandyopadhyay, S. Roy, T. Ueda, and K. Hasuike, "Multipath routing in ad hoc networks with directional antenna," in Proc. IFIP Conf. PWC, Oct. 2002, pp. 45-52.

[5] J. Tang, G. Xue, C. Chandler, and W. Zhang, "Interference-aware routing in multihop wireless networks using directional antennas," in Proc. IEEE INFOCOM, Mar. 2005, pp. 751-760.

[6] H. Zhai, X. Chen, and Y. Fang, "Alleviating intra-flow and inter-flow contentions for reliable service in mobile ad hoc networks," in Proc. IEEE MILCOM, Nov. 2004, pp. 1640-1646.

[7] H. Zhai and Y. Fang, "Distributed flow control and medium access in multihop ad hoc networks," IEEE Trans. Mobile Comput., vol. 5, no. 11, pp. 1503-1514, Nov. 2006.

[8] H. Zhai, J. Wang, and Y. Fang, "DUCHA: A new dual-channel MAC protocol for multihop ad hoc networks," IEEE Trans. Wireless Commun., vol. 5, no. 11, pp. 3224-3233, Nov. 2006.

[9] H. Zhai, X. Chen, and Y. Fang, "Improving transport layer performance in multihop ad hoc networks by exploiting MAC layer information," IEEE Trans. Wireless Commun., vol. 6, no. 5, pp. 1692-1701, May 2007.

[10] W. Wei and A. Zakhor, "Robust multipath source routing protocol (RMPSR) video communication over wireless ad hoc networks," in Proc. IEEE Int. Conf. Multimedia Expo., Jun. 2004, pp. 1379-1382.

[11] S. J. Lee and M. Gerla, "Split multipath routing with maximally disjoint paths in ad hoc networks," in Proc. IEEE ICC, 2001, pp. 3201-3205.

[12] Z. Ye, S. V. Krishnamurthy, and S. K. Tripathi, "A framework for reliable routing in mobile ad hoc networks," in Proc. IEEE INFOCOM, Apr. 2003, pp. 270-280.

[13] M. K. Marina and S. R. Das, "On-demand multipath distance vector routing in ad hoc networks," in Proc. IEEE ICNP, Nov. 2001, pp. 14-23.

[14] X. Li and D. Cuthbert, "Node-disjointness-based multipath routing for mobile ad hoc networks," in Proc. PE-WASUN, Oct. 2004, pp. 23-29.

[15] A. Nasipuri, R. Castañeda, and S. R. Das, "Performance of multipath routing for on-demand protocols in mobile ad hoc networks," Mobile Netw. Appl., vol. 6, no. 4, pp. 339-349, Aug. 2001.

[16] A. C. Valera, W. K. G. Seah, and S. V. Rao, "Improving protocol robustness in ad hoc networks through cooperative packet caching and shortest multipath routing," IEEE Trans. Mobile Comput., vol. 4, no. 5, pp. 443457, Sep./Oct. 2005.

[17] V. Raghunathan and P. R. Kumar, "Issues in Wardrop routing in wireless networks," in Proc. IEEE WICON, Jul. 2005, pp. 34-41.

[18] B. Deb, S. Bhatnagar, and B. Nath, "ReInForM: Reliable information forwarding using multiple paths in sensor networks," in Proc. Int. Conf. Local Comput. Netw., Oct. 2003, pp. 406-415.

[19] A. Tsirigos and Z. J. Hass, "Analysis of multipath routing-Part 2: Mitigation of the effects of frequently changing network topologies," IEEE Trans. Wireless Commun., vol. 3, no. 2, pp. 500-511, Mar. 2004.

[20] A. Orda and A. Sprintson, "Efficient algorithms for computing disjoint QoS paths," in Proc. IEEE INFOCOM, Mar. 2004, pp. 727-738.

[21] E. Felemban, C. G. Lee, R. Boder, and S. Vural, "Probabilistic QoS guarantee in reliability and timeliness domains in wireless sensor networks," in Proc. IEEE INFOCOM, Mar. 2005, pp. 2646-2657.

[22] K. Jain, J. Padhye, V. Padmanabhan, and L. Qiu, "Impact of interference on multi-hop wireless network performance," in Proc. ACM MOBICOM, Sep. 2003, pp. 66-80.

Xiaoxia Huang, photograph and biography not available at the time of publication.

Yuguang "Michael" Fang, photograph and biography not available at the time of publication. 